Acta Crystallographica Section E

Structure Reports

Online

ISSN 1600-5368

\section{2-[4-(4-Methylphenylsulfonyl)piperazin- 1-yl]-1-(4,5,6,7-tetrahydrothieno[3,2-c]- pyridin-5-yl)ethanone}

\section{Duan Niu, ${ }^{a}$ Shu-Yun Huang, ${ }^{b}$ Ping-Bao Wang ${ }^{b}$ and Deng-Ke Liu ${ }^{\text {b* }}$}

aTianjin Medical University, Tianjin 300070, People's Republic of China, and

bianjin Institute of Pharmaceutical Research, Tianjin, 300193, People's Republic of China

Correspondence e-mail: liudk@tjipr.com

Received 27 June 2011; accepted 17 July 2011

Key indicators: single-crystal X-ray study; $T=113 \mathrm{~K}$; mean $\sigma(\mathrm{C}-\mathrm{C})=0.003 \AA$; $R$ factor $=0.054 ; w R$ factor $=0.143 ;$ data-to-parameter ratio $=19.1$.

In the title thienopyridine derivative, $\mathrm{C}_{20} \mathrm{H}_{25} \mathrm{~N}_{3} \mathrm{O}_{3} \mathrm{~S}_{2}$, the piperazine ring exhibits a chair conformation and the tetrahydropyridine ring exhibits a half-chair conformation. The folded conformation of the molecule is defined by the $\mathrm{N}-\mathrm{C}-$ $\mathrm{C}-\mathrm{N}$ torsion angle of -70.20 (2) ${ }^{\circ}$. Intermolecular $\mathrm{C}-\mathrm{H} \cdots \mathrm{S}$ and $\mathrm{C}-\mathrm{H} \cdots \mathrm{O}$ hydrogen bonds help to establish the packing.

\section{Related literature}

For background to the bioactivity and applications of the title compound, see: Cattaneo (2009); Wallentin (2009). For a related structure, see: Zhi et al. (2011). For the synthesis of the title compound, see: Liu et al. (2008).

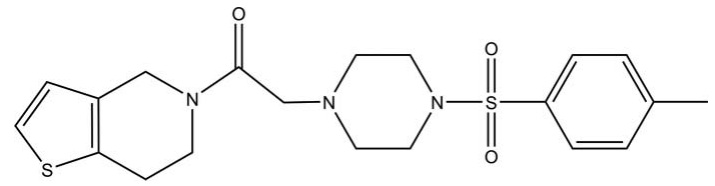

\section{Experimental}

Crystal data

$\mathrm{C}_{20} \mathrm{H}_{25} \mathrm{~N}_{3} \mathrm{O}_{3} \mathrm{~S}_{2}$

$M_{r}=419.55$ $b=15.710$ (3) $\AA$

$c=19.798$ (3) $\AA$

$V=4062.8(11) \AA^{3}$

$Z=8$

Data collection

Rigaku Saturn CCD area-detector diffractometer

Absorption correction: multi-scan (CrystalClear; Rigaku/MSC, 2005)

$T_{\min }=0.934, T_{\max }=0.950$

Refinement

$R\left[F^{2}>2 \sigma\left(F^{2}\right)\right]=0.054$

$w R\left(F^{2}\right)=0.143$

$S=1.14$

4844 reflections
Mo $K \alpha$ radiation

$\mu=0.29 \mathrm{~mm}^{-1}$

$T=113 \mathrm{~K}$

$0.24 \times 0.20 \times 0.18 \mathrm{~mm}$

49454 measured reflections 4844 independent reflections 4463 reflections with $I>2 \sigma(I)$ $R_{\text {int }}=0.054$

Table 1

Hydrogen-bond geometry $\left(\AA{ }^{\circ}\right)$.

\begin{tabular}{llllll}
\hline$D-\mathrm{H} \cdots A$ & $D-\mathrm{H}$ & $\mathrm{H} \cdots A$ & $D \cdots A$ & \multicolumn{1}{c}{$D-\mathrm{H} \cdots A$} \\
\hline $\mathrm{C} 5-\mathrm{H} 5 A \cdots \mathrm{S} 1^{\mathrm{i}}$ & 0.99 & 2.77 & $3.469(2)$ & 128 \\
$\mathrm{C} 6-\mathrm{H} 6 A \cdots \mathrm{O} 1^{\mathrm{ii}}$ & 0.99 & 2.52 & $3.470(3)$ & 161 & \\
$\mathrm{C} 6-\mathrm{H} 6 B \cdots \mathrm{O} 2^{\text {ii }}$ & 0.99 & 2.51 & $3.346(3)$ & 143 & \\
\hline Symmetry codes: & $(\mathrm{i})$ & $x+\frac{1}{2},-y+\frac{3}{2},-z+1 ;$ & (ii) & $-x,-y+2,-z+1 ;$ & (iii) \\
$-x+\frac{1}{2}, y-\frac{1}{2}, z$. & & & & &
\end{tabular}

Data collection: CrystalClear (Rigaku/MSC, 2005); cell refinement: CrystalClear; data reduction: CrystalClear; program(s) used to solve structure: SHELXS97 (Sheldrick, 2008); program(s) used to refine structure: SHELXL97 (Sheldrick, 2008); molecular graphics: SHELXTL (Sheldrick, 2008); software used to prepare material for publication: CrystalStructure (Rigaku/MSC, 2005).

The authors thank Mr Hai-Bin Song of Nankai University for the X-ray crystallographic determinations and helpful suggestions.

Supplementary data and figures for this paper are available from the IUCr electronic archives (Reference: FL2350).

\title{
References
}

Cattaneo, M. (2009). J. Thromb. Haemost. 7, Suppl. 1, 262-265.

Liu, D. K., Liu, Y., Liu, M., Zhang, S. J., Cheng, D., Jin, L. Y., Xu, W. R. \& Liu, C. X. (2008). CN Patent 101284838A.

Rigaku/MSC (2005). CrystalClear and CrystalStructure. Rigaku/MSC Inc., The Woodlands, Texas, USA.

Sheldrick, G. M. (2008). Acta Cryst. A64, 112-122.

Wallentin, L. (2009). Eur. Heart J. 30, 1964-1977.

Zhi, S., Mu, S., Liu, Y. \& Liu, D.-K. (2011). Acta Cryst. E67, o1490. 


\section{supporting information}

Acta Cryst. (2011). E67, o2134 [doi:10.1107/S1600536811028716]

\section{2-[4-(4-Methylphenylsulfonyl)piperazin-1-yl]-1-(4,5,6,7-tetrahydrothieno[3,2- c]pyridin-5-yl)ethanone}

\section{Duan Niu, Shu-Yun Huang, Ping-Bao Wang and Deng-Ke Liu}

\section{S1. Comment}

As a thienopyridine derivative, the title compound(I) can be used as an irreversible P2Y12 antagonist to inhibit ADP, which induces platelet aggregation and decreases the risk of arterial occlusion. (Cattaneo 2009; Wallentin 2009).

The piperazine ring exhibits a chair conformation and the tetrahydropyridine ring exhibits a half chair conformation (Fig. 1). The folded conformation of the molecule is defined by the $\mathrm{N} 1-\mathrm{C} 8-\mathrm{C} 9-\mathrm{N} 2$ torsion angle of $-70.20(2)^{\circ}$. The dihedral angles formed between the tetrahydropyridine plane and the phenyl ring and the $\mathrm{C} 10-\mathrm{C} 11-\mathrm{C} 12-\mathrm{C} 13$ plane are $85.47(6)^{\circ}$ and $56.38(9)^{\circ}$, respectively. The crystal is stabilized by intermolecular $\mathrm{C}-\mathrm{H} \cdots \mathrm{S}$ and $\mathrm{C}-\mathrm{H} \cdots \mathrm{O}$ hydrogen bonds (Table1, Fig.2).

\section{S2. Experimental}

2-Chloroacetyl chloride was added dropwise into a mixture of 4,5,6,7-tetrahydrothieno[3,2-c]pyridine, dichloromethane and TEA at 263k-273k. After stirring for $3 \mathrm{~h}$, the solvent was evaporated and a light yellow oily substance was obtained by silica gel column chromatography. The light yellow oily substance was then dissloved in a mixture of acetonitrile, TEA and 1-tosylpiperazine. After stirring for $5 \mathrm{~h}$, the title compound was obtained by silica gel column chromatography. Crystallization of the resultingg white solid from methanol afforded white crystals suitble for X-ray analysis.

\section{S3. Refinement}

The $\mathrm{H}$ atoms were positioned geometrioncally and refined using a riding model with $\mathrm{d}(\mathrm{C}-\mathrm{H})=0.95-0.99 \AA$, and $U_{\text {iso }}(\mathrm{H})=1.2 U_{\text {eq }}(\mathrm{CH}$ and $\mathrm{CH} 2)$ or $1.5 U_{\mathrm{eq}}(\mathrm{CH} 3)$ of the parent atom. 


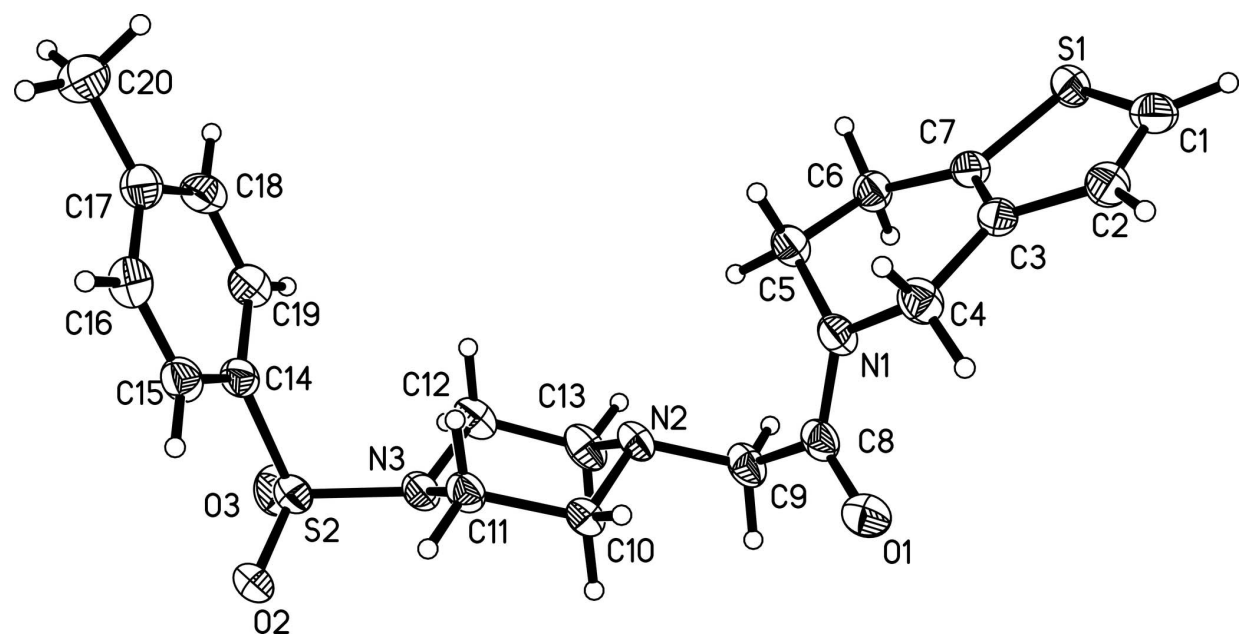

\section{Figure 1}

The molecular structure of (I), with the atom-numbering scheme and $50 \%$ probability displacement ellipsoids.

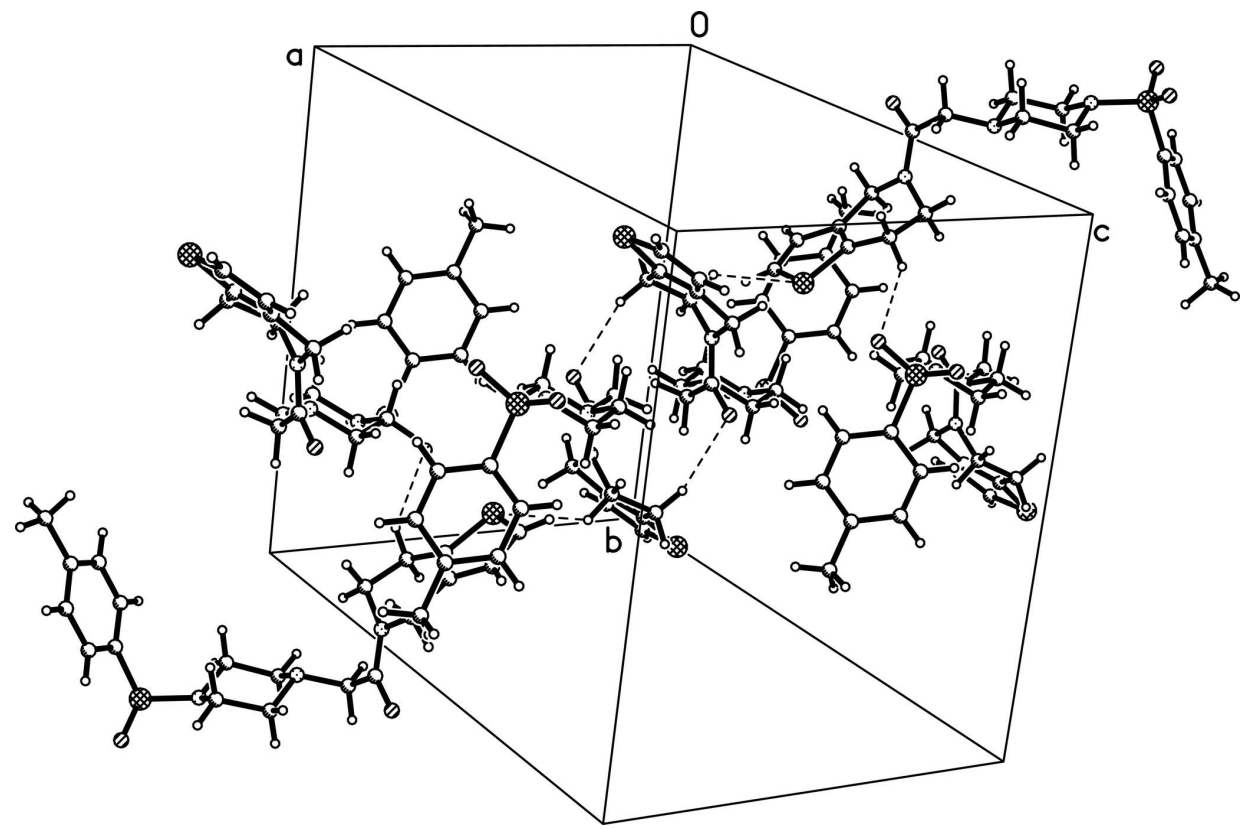

Figure 2

Packing diagram for (I) with hydrogen bonds drawn as dashed lines.

\section{2-[4-(4-Methylphenylsulfonyl)piperazin-1-yl]-1-(4,5,6,7- tetrahydrothieno[3,2-c]pyridin-5-yl)ethanone}

\section{Crystal data}

\section{$\mathrm{C}_{20} \mathrm{H}_{25} \mathrm{~N}_{3} \mathrm{O}_{3} \mathrm{~S}_{2}$}

$M_{r}=419.55$

Orthorhombic, $\mathrm{Pbca}$

Hall symbol: -P $2 \mathrm{ac} 2 \mathrm{ab}$

$a=13.062(2) \AA$

$b=15.710(3) \AA$

$c=19.798(3) \AA$

$V=4062.8(11) \AA^{3}$

$Z=8$
$F(000)=1776$

$D_{\mathrm{x}}=1.372 \mathrm{Mg} \mathrm{m}^{-3}$

Mo $K \alpha$ radiation, $\lambda=0.71073 \AA$

Cell parameters from 12748 reflections

$\theta=1.7-28.0^{\circ}$

$\mu=0.29 \mathrm{~mm}^{-1}$

$T=113 \mathrm{~K}$

Prism, colorless

$0.24 \times 0.20 \times 0.18 \mathrm{~mm}$ 


\section{Data collection}

Rigaku Saturn CCD area-detector diffractometer

Radiation source: rotating anode

Multilayer monochromator

Detector resolution: 14.63 pixels $\mathrm{mm}^{-1}$

$\omega$ and $\varphi$ scans

Absorption correction: multi-scan CrystalClear

$T_{\min }=0.934, T_{\max }=0.950$

Refinement

Refinement on $F^{2}$

Least-squares matrix: full

$R\left[F^{2}>2 \sigma\left(F^{2}\right)\right]=0.054$

$w R\left(F^{2}\right)=0.143$

$S=1.14$

4844 reflections

254 parameters

0 restraints

Primary atom site location: structure-invariant direct methods
49454 measured reflections

4844 independent reflections

4463 reflections with $I>2 \sigma(I)$

$R_{\text {int }}=0.054$

$\theta_{\max }=27.9^{\circ}, \theta_{\min }=2.1^{\circ}$

$h=-17 \rightarrow 17$

$k=-20 \rightarrow 20$

$l=-26 \rightarrow 25$
Secondary atom site location: difference Fourier map

Hydrogen site location: inferred from neighbouring sites

$\mathrm{H}$-atom parameters constrained

$w=1 /\left[\sigma^{2}\left(F_{\mathrm{o}}^{2}\right)+(0.0665 P)^{2}+2.3859 P\right]$

where $P=\left(F_{\mathrm{o}}^{2}+2 F_{\mathrm{c}}^{2}\right) / 3$

$(\Delta / \sigma)_{\max }=0.002$

$\Delta \rho_{\max }=1.17 \mathrm{e} \AA^{-3}$

$\Delta \rho_{\min }=-0.34$ e $\AA^{-3}$

\section{Special details}

Geometry. All e.s.d.'s (except the e.s.d. in the dihedral angle between two 1.s. planes) are estimated using the full covariance matrix. The cell e.s.d.'s are taken into account individually in the estimation of e.s.d.'s in distances, angles and torsion angles; correlations between e.s.d.'s in cell parameters are only used when they are defined by crystal symmetry. An approximate (isotropic) treatment of cell e.s.d.'s is used for estimating e.s.d.'s involving 1.s. planes.

Refinement. Refinement of $F^{2}$ against ALL reflections. The weighted $R$-factor $w R$ and goodness of fit $S$ are based on $F^{2}$, conventional $R$-factors $R$ are based on $F$, with $F$ set to zero for negative $F^{2}$. The threshold expression of $F^{2}>\sigma\left(F^{2}\right)$ is used only for calculating $R$-factors $(\mathrm{gt})$ etc. and is not relevant to the choice of reflections for refinement. $R$-factors based on $F^{2}$ are statistically about twice as large as those based on $F$, and $R$-factors based on ALL data will be even larger.

Fractional atomic coordinates and isotropic or equivalent isotropic displacement parameters $\left(\AA^{2}\right)$

\begin{tabular}{lllll}
\hline & $x$ & $y$ & $z$ & $U_{\text {iso }} * / U_{\text {eq }}$ \\
\hline S1 & $-0.12585(4)$ & $0.69571(4)$ & $0.50761(3)$ & $0.03055(16)$ \\
S2 & $0.51658(4)$ & $1.09372(3)$ & $0.30610(3)$ & $0.03065(16)$ \\
O1 & $0.13122(12)$ & $1.01184(10)$ & $0.57588(8)$ & $0.0348(4)$ \\
O2 & $0.58272(13)$ & $1.14367(10)$ & $0.34791(9)$ & $0.0400(4)$ \\
O3 & $0.48630(14)$ & $1.12484(11)$ & $0.24122(9)$ & $0.0420(4)$ \\
N1 & $0.12333(13)$ & $0.89002(11)$ & $0.51535(9)$ & $0.0273(4)$ \\
N2 & $0.23688(12)$ & $1.01546(10)$ & $0.41984(8)$ & $0.0244(4)$ \\
N3 & $0.41057(13)$ & $1.07905(11)$ & $0.34905(9)$ & $0.0271(4)$ \\
C1 & $-0.11495(17)$ & $0.69019(14)$ & $0.59285(13)$ & $0.0329(5)$ \\
H1 & -0.1601 & 0.6579 & 0.6204 & $0.039 *$ \\
C2 & $-0.03617(16)$ & $0.73600(13)$ & $0.61798(11)$ & $0.0293(4)$ \\
H2 & -0.0186 & 0.7390 & 0.6645 & $0.035^{*}$ \\
C3 & $0.01696(15)$ & $0.77916(12)$ & $0.56530(10)$ & $0.0240(4)$ \\
C4 & $0.10712(17)$ & $0.83828(14)$ & $0.57598(11)$ & $0.0306(5)$ \\
H4A & 0.0937 & 0.8758 & 0.6152 & $0.037^{*}$ \\
H4B & 0.1694 & 0.8045 & 0.5857 & $0.037^{*}$
\end{tabular}




$\begin{array}{lllll}\text { C5 } & 0.11965(16) & 0.84157(14) & 0.45193(11) & 0.0278(4) \\ \text { H5A } & 0.1705 & 0.7949 & 0.4538 & 0.033^{*} \\ \text { H5B } & 0.1384 & 0.8795 & 0.4139 & 0.033^{*} \\ \text { C6 } & 0.01385(16) & 0.80409(14) & 0.43904(10) & 0.0277(4) \\ \text { H6A } & -0.0342 & 0.8495 & 0.4249 & 0.033^{*} \\ \text { H6B } & 0.0174 & 0.7610 & 0.4026 & 0.033^{*} \\ \text { C7 } & -0.02224(15) & 0.76393(13) & 0.50316(11) & 0.0252(4) \\ \text { C8 } & 0.13226(14) & 0.97516(13) & 0.52092(11) & 0.0266(4) \\ \text { C9 } & 0.13997(15) & 1.02709(14) & 0.45566(12) & 0.0302(5) \\ \text { H9A } & 0.0831 & 1.0106 & 0.4253 & 0.036^{*} \\ \text { H9B } & 0.1316 & 1.0882 & 0.4667 & 0.036^{*} \\ \text { C10 } & 0.32177(15) & 1.05628(13) & 0.45626(11) & 0.0260(4) \\ \text { H10A } & 0.3281 & 1.0309 & 0.5018 & 0.031^{*} \\ \text { H10B } & 0.3072 & 1.1177 & 0.4617 & 0.031^{*} \\ \text { C11 } & 0.42175(15) & 1.04500(14) & 0.41822(10) & 0.0264(4) \\ \text { H11A } & 0.4774 & 1.0755 & 0.4420 & 0.032^{*} \\ \text { H11B } & 0.4399 & 0.9839 & 0.4163 & 0.032^{*} \\ \text { C12 } & 0.32501(17) & 1.03841(14) & 0.31276(11) & 0.0306(5) \\ \text { H12A } & 0.3378 & 0.9766 & 0.3081 & 0.037^{*} \\ \text { H12B } & 0.3183 & 1.0632 & 0.2670 & 0.037^{*} \\ \text { C13 } & 0.22808(17) & 1.05332(15) & 0.35261(11) & 0.0321(5) \\ \text { H13A } & 0.2155 & 1.1152 & 0.3568 & 0.039^{*} \\ \text { H13B } & 0.1693 & 1.0278 & 0.3285 & 0.039^{*} \\ \text { C14 } & 0.57571(17) & 0.99377(13) & 0.29458(10) & 0.0273(4) \\ \text { C15 } & 0.66141(17) & 0.97308(14) & 0.33228(11) & 0.0305(5) \\ \text { H15 } & 0.6860 & 1.0112 & 0.3658 & 0.037^{*} \\ \text { C16 } & 0.71122(18) & 0.89631(15) & 0.32094(12) & 0.0344(5) \\ \text { H16 } & 0.7705 & 0.8825 & 0.3465 & 0.041^{*} \\ \text { C17 } & 0.67544(18) & 0.83934(14) & 0.27257(11) & 0.0335(5) \\ \text { C18 } & 0.58779(19) & 0.86056(14) & 0.23633(11) & 0.0334(5) \\ \text { H18 } & 0.5617 & 0.8215 & 0.2040 & 0.040^{*} \\ \text { C19 } & 0.53773(18) & 0.93727(14) & 0.24632(11) & 0.0304(5) \\ \text { H19 } & 0.4785 & 0.9512 & 0.2207 & 0.037^{*} \\ \text { C20 } & 0.7307(2) & 0.75663(16) & 0.25933(14) & 0.0474(6) \\ \text { H20A } & 0.7981 & 0.7581 & 0.2810 & 0.071^{*} \\ \text { H20B } & 0.7392 & 0.7488 & 0.2105 & 0.071^{*} \\ \text { H20C } & 0.6907 & 0.7093 & 0.2778 & 0.071^{*} \\ & & & & \end{array}$

Atomic displacement parameters $\left(\AA^{2}\right)$

\begin{tabular}{lllllll}
\hline & $U^{11}$ & $U^{22}$ & $U^{33}$ & $U^{12}$ & $U^{13}$ & $U^{23}$ \\
\hline S1 & $0.0261(3)$ & $0.0310(3)$ & $0.0345(3)$ & $-0.0073(2)$ & $0.0029(2)$ & $-0.0032(2)$ \\
S2 & $0.0320(3)$ & $0.0252(3)$ & $0.0348(3)$ & $-0.0070(2)$ & $0.0075(2)$ & $-0.0003(2)$ \\
O1 & $0.0353(9)$ & $0.0313(8)$ & $0.0377(9)$ & $-0.0053(7)$ & $-0.0024(7)$ & $-0.0032(7)$ \\
O2 & $0.0348(9)$ & $0.0328(8)$ & $0.0523(10)$ & $-0.0150(7)$ & $0.0129(7)$ & $-0.0125(7)$ \\
O3 & $0.0482(10)$ & $0.0385(9)$ & $0.0391(9)$ & $-0.0008(8)$ & $0.0106(8)$ & $0.0138(8)$ \\
N1 & $0.0287(9)$ & $0.0259(9)$ & $0.0274(9)$ & $-0.0068(7)$ & $0.0013(7)$ & $0.0031(7)$ \\
N2 & $0.0197(8)$ & $0.0239(8)$ & $0.0295(9)$ & $-0.0049(6)$ & $-0.0029(6)$ & $0.0043(7)$
\end{tabular}


supporting information

$\begin{array}{lllllll}\mathrm{N} 3 & 0.0251(9) & 0.0267(9) & 0.0296(9) & -0.0061(7) & 0.0007(7) & 0.0000(7) \\ \text { C1 } & 0.0266(11) & 0.0255(10) & 0.0466(13) & 0.0001(8) & 0.0003(9) & -0.0044(9) \\ \text { C2 } & 0.0326(11) & 0.0271(10) & 0.0281(11) & 0.0028(9) & 0.0004(8) & 0.0047(8) \\ \text { C3 } & 0.0261(10) & 0.0202(9) & 0.0258(10) & 0.0021(7) & 0.0017(8) & 0.0020(7) \\ \text { C4 } & 0.0348(11) & 0.0304(11) & 0.0266(11) & -0.0048(9) & -0.0019(8) & 0.0035(9) \\ \text { C5 } & 0.0266(10) & 0.0281(10) & 0.0287(11) & -0.0057(8) & 0.0007(8) & 0.0012(8) \\ \text { C6 } & 0.0290(10) & 0.0304(11) & 0.0238(10) & -0.0041(8) & 0.0012(8) & -0.0015(8) \\ \text { C7 } & 0.0246(10) & 0.0219(9) & 0.0290(10) & 0.0001(8) & 0.0009(8) & -0.0023(8) \\ \text { C8 } & 0.0159(9) & 0.0266(10) & 0.0372(12) & -0.0027(7) & -0.0010(8) & 0.0012(9) \\ \text { C9 } & 0.0197(9) & 0.0280(10) & 0.0428(13) & -0.0018(8) & -0.0005(8) & 0.0067(9) \\ \text { C10 } & 0.0225(9) & 0.0252(9) & 0.0302(10) & -0.0060(8) & -0.0009(8) & -0.0014(8) \\ \text { C11 } & 0.0219(9) & 0.0288(10) & 0.0286(10) & -0.0053(8) & -0.0025(8) & -0.0017(8) \\ \text { C12 } & 0.0328(11) & 0.0309(11) & 0.0282(11) & -0.0097(9) & -0.0051(8) & 0.0040(8) \\ \text { C13 } & 0.0269(10) & 0.0340(11) & 0.0356(12) & -0.0071(9) & -0.0075(9) & 0.0110(9) \\ \text { C14 } & 0.0315(11) & 0.0273(10) & 0.0231(10) & -0.0076(8) & 0.0059(8) & -0.0021(8) \\ \text { C15 } & 0.0307(11) & 0.0356(11) & 0.0251(10) & -0.0085(9) & 0.0022(8) & -0.0038(9) \\ \text { C16 } & 0.0311(11) & 0.0380(12) & 0.0342(12) & -0.0022(9) & 0.0022(9) & 0.0020(9) \\ \text { C17 } & 0.0385(12) & 0.0306(11) & 0.0316(11) & -0.0045(9) & 0.0118(9) & 0.0007(9) \\ \text { C18 } & 0.0467(13) & 0.0304(11) & 0.0230(10) & -0.0113(10) & 0.0065(9) & -0.0045(8) \\ \text { C19 } & 0.0355(11) & 0.0332(11) & 0.0225(10) & -0.0075(9) & -0.0003(8) & 0.0002(8) \\ \text { C20 } & 0.0517(16) & 0.0343(12) & 0.0561(16) & 0.0025(11) & 0.0162(13) & -0.0035(12)\end{array}$

Geometric parameters $\left(\AA,{ }^{\circ}\right)$

\begin{tabular}{llll}
\hline $\mathrm{S} 1-\mathrm{C} 1$ & $1.696(3)$ & $\mathrm{C} 6-\mathrm{H} 6 \mathrm{~B}$ & 0.9900 \\
$\mathrm{~S} 1-\mathrm{C} 7$ & $1.729(2)$ & $\mathrm{C} 8-\mathrm{C} 9$ & $1.531(3)$ \\
$\mathrm{S} 2-\mathrm{O} 3$ & $1.4302(18)$ & $\mathrm{C} 9-\mathrm{H} 9 \mathrm{~A}$ & 0.9900 \\
$\mathrm{~S} 2-\mathrm{O} 2$ & $1.4309(17)$ & $\mathrm{C} 9-\mathrm{H} 9 \mathrm{~B}$ & 0.9900 \\
$\mathrm{~S} 2-\mathrm{N} 3$ & $1.6412(18)$ & $\mathrm{C} 10-\mathrm{C} 11$ & $1.518(3)$ \\
$\mathrm{S} 2-\mathrm{C} 14$ & $1.765(2)$ & $\mathrm{C} 10-\mathrm{H} 10 \mathrm{~A}$ & 0.9900 \\
$\mathrm{O} 1-\mathrm{C} 8$ & $1.231(3)$ & $\mathrm{C} 10-\mathrm{H} 10 \mathrm{~B}$ & 0.9900 \\
$\mathrm{~N} 1-\mathrm{C} 8$ & $1.347(3)$ & $\mathrm{C} 11-\mathrm{H} 11 \mathrm{~A}$ & 0.9900 \\
$\mathrm{~N} 1-\mathrm{C} 4$ & $1.465(3)$ & $\mathrm{C} 11-\mathrm{H} 11 \mathrm{~B}$ & 0.9900 \\
$\mathrm{~N} 1-\mathrm{C} 5$ & $1.469(3)$ & $\mathrm{C} 12-\mathrm{C} 13$ & $1.510(3)$ \\
$\mathrm{N} 2-\mathrm{C} 9$ & $1.462(3)$ & $\mathrm{C} 12-\mathrm{H} 12 \mathrm{~A}$ & 0.9900 \\
$\mathrm{~N} 2-\mathrm{C} 13$ & $1.463(3)$ & $\mathrm{C} 12-\mathrm{H} 12 \mathrm{~B}$ & 0.9900 \\
$\mathrm{~N} 2-\mathrm{C} 10$ & $1.470(2)$ & $\mathrm{C} 13-\mathrm{H} 13 \mathrm{~A}$ & 0.9900 \\
$\mathrm{~N} 3-\mathrm{C} 12$ & $1.474(3)$ & $\mathrm{C} 13-\mathrm{H} 13 \mathrm{~B}$ & 0.9900 \\
$\mathrm{~N} 3-\mathrm{C} 11$ & $1.478(3)$ & $\mathrm{C} 14-\mathrm{C} 15$ & $1.384(3)$ \\
$\mathrm{C} 1-\mathrm{C} 2$ & $1.351(3)$ & $\mathrm{C} 14-\mathrm{C} 19$ & $1.395(3)$ \\
$\mathrm{C} 1-\mathrm{H} 1$ & 0.9500 & $\mathrm{C} 15-\mathrm{C} 16$ & $1.389(3)$ \\
$\mathrm{C} 2-\mathrm{C} 3$ & $1.424(3)$ & $\mathrm{C} 15-\mathrm{H} 15$ & 0.9500 \\
$\mathrm{C} 2-\mathrm{H} 2$ & 0.9500 & $\mathrm{C} 16-\mathrm{C} 17$ & $1.392(3)$ \\
$\mathrm{C} 3-\mathrm{C} 7$ & $1.354(3)$ & $\mathrm{C} 16-\mathrm{H} 16$ & 0.9500 \\
$\mathrm{C} 3-\mathrm{C} 4$ & $1.515(3)$ & $\mathrm{C} 17-\mathrm{C} 18$ & $1.392(3)$ \\
$\mathrm{C} 4-\mathrm{H} 4 \mathrm{~A}$ & 0.9900 & $\mathrm{C} 17-\mathrm{C} 20$ & $1.510(3)$ \\
$\mathrm{C} 4-\mathrm{H} 4 \mathrm{~B}$ & 0.9900 & $\mathrm{C} 18-\mathrm{C} 19$ & $1.385(3)$ \\
$\mathrm{C} 5-\mathrm{C} 6$ & $1.524(3)$ & $\mathrm{C} 18-\mathrm{H} 18$ & 0.9500 \\
& & &
\end{tabular}




\begin{tabular}{|c|c|c|c|}
\hline C5-H5A & 0.9900 & $\mathrm{C} 19-\mathrm{H} 19$ & 0.9500 \\
\hline C5-H5B & 0.9900 & $\mathrm{C} 20-\mathrm{H} 20 \mathrm{~A}$ & 0.9800 \\
\hline $\mathrm{C} 6-\mathrm{C} 7$ & $1.494(3)$ & $\mathrm{C} 20-\mathrm{H} 20 \mathrm{~B}$ & 0.9800 \\
\hline $\mathrm{C} 6-\mathrm{H} 6 \mathrm{~A}$ & 0.9900 & $\mathrm{C} 20-\mathrm{H} 20 \mathrm{C}$ & 0.9800 \\
\hline $\mathrm{C} 1-\mathrm{S} 1-\mathrm{C} 7$ & $90.96(11)$ & $\mathrm{N} 2-\mathrm{C} 9-\mathrm{H} 9 \mathrm{~B}$ & 108.9 \\
\hline $\mathrm{O} 3-\mathrm{S} 2-\mathrm{O} 2$ & $119.94(11)$ & $\mathrm{C} 8-\mathrm{C} 9-\mathrm{H} 9 \mathrm{~B}$ & 108.9 \\
\hline $\mathrm{O} 3-\mathrm{S} 2-\mathrm{N} 3$ & $106.26(10)$ & $\mathrm{H} 9 \mathrm{~A}-\mathrm{C} 9-\mathrm{H} 9 \mathrm{~B}$ & 107.7 \\
\hline $\mathrm{O} 2-\mathrm{S} 2-\mathrm{N} 3$ & $106.67(9)$ & $\mathrm{N} 2-\mathrm{C} 10-\mathrm{C} 11$ & $110.78(16)$ \\
\hline $\mathrm{O} 3-\mathrm{S} 2-\mathrm{C} 14$ & $108.00(10)$ & $\mathrm{N} 2-\mathrm{C} 10-\mathrm{H} 10 \mathrm{~A}$ & 109.5 \\
\hline $\mathrm{O} 2-\mathrm{S} 2-\mathrm{C} 14$ & $107.37(11)$ & $\mathrm{C} 11-\mathrm{C} 10-\mathrm{H} 10 \mathrm{~A}$ & 109.5 \\
\hline N3-S2-C14 & $108.14(9)$ & $\mathrm{N} 2-\mathrm{C} 10-\mathrm{H} 10 \mathrm{~B}$ & 109.5 \\
\hline $\mathrm{C} 8-\mathrm{N} 1-\mathrm{C} 4$ & $119.75(18)$ & $\mathrm{C} 11-\mathrm{C} 10-\mathrm{H} 10 \mathrm{~B}$ & 109.5 \\
\hline $\mathrm{C} 8-\mathrm{N} 1-\mathrm{C} 5$ & $125.96(18)$ & $\mathrm{H} 10 \mathrm{~A}-\mathrm{C} 10-\mathrm{H} 10 \mathrm{~B}$ & 108.1 \\
\hline $\mathrm{C} 4-\mathrm{N} 1-\mathrm{C} 5$ & $114.08(17)$ & $\mathrm{N} 3-\mathrm{C} 11-\mathrm{C} 10$ & $109.42(17)$ \\
\hline $\mathrm{C} 9-\mathrm{N} 2-\mathrm{C} 13$ & $108.82(16)$ & $\mathrm{N} 3-\mathrm{C} 11-\mathrm{H} 11 \mathrm{~A}$ & 109.8 \\
\hline $\mathrm{C} 9-\mathrm{N} 2-\mathrm{C} 10$ & $111.13(16)$ & $\mathrm{C} 10-\mathrm{C} 11-\mathrm{H} 11 \mathrm{~A}$ & 109.8 \\
\hline $\mathrm{C} 13-\mathrm{N} 2-\mathrm{C} 10$ & $109.16(15)$ & N3-C11-H11B & 109.8 \\
\hline $\mathrm{C} 12-\mathrm{N} 3-\mathrm{C} 11$ & $111.71(16)$ & $\mathrm{C} 10-\mathrm{C} 11-\mathrm{H} 11 \mathrm{~B}$ & 109.8 \\
\hline $\mathrm{C} 12-\mathrm{N} 3-\mathrm{S} 2$ & $116.61(14)$ & $\mathrm{H} 11 \mathrm{~A}-\mathrm{C} 11-\mathrm{H} 11 \mathrm{~B}$ & 108.2 \\
\hline $\mathrm{C} 11-\mathrm{N} 3-\mathrm{S} 2$ & $116.59(14)$ & $\mathrm{N} 3-\mathrm{C} 12-\mathrm{C} 13$ & $108.29(18)$ \\
\hline $\mathrm{C} 2-\mathrm{C} 1-\mathrm{S} 1$ & $113.78(18)$ & $\mathrm{N} 3-\mathrm{C} 12-\mathrm{H} 12 \mathrm{~A}$ & 110.0 \\
\hline $\mathrm{C} 2-\mathrm{C} 1-\mathrm{H} 1$ & 123.1 & $\mathrm{C} 13-\mathrm{C} 12-\mathrm{H} 12 \mathrm{~A}$ & 110.0 \\
\hline $\mathrm{S} 1-\mathrm{C} 1-\mathrm{H} 1$ & 123.1 & $\mathrm{~N} 3-\mathrm{C} 12-\mathrm{H} 12 \mathrm{~B}$ & 110.0 \\
\hline $\mathrm{C} 1-\mathrm{C} 2-\mathrm{C} 3$ & $110.8(2)$ & $\mathrm{C} 13-\mathrm{C} 12-\mathrm{H} 12 \mathrm{~B}$ & 110.0 \\
\hline $\mathrm{C} 1-\mathrm{C} 2-\mathrm{H} 2$ & 124.6 & $\mathrm{H} 12 \mathrm{~A}-\mathrm{C} 12-\mathrm{H} 12 \mathrm{~B}$ & 108.4 \\
\hline $\mathrm{C} 3-\mathrm{C} 2-\mathrm{H} 2$ & 124.6 & $\mathrm{~N} 2-\mathrm{C} 13-\mathrm{C} 12$ & $110.27(17)$ \\
\hline $\mathrm{C} 7-\mathrm{C} 3-\mathrm{C} 2$ & $113.39(19)$ & $\mathrm{N} 2-\mathrm{C} 13-\mathrm{H} 13 \mathrm{~A}$ & 109.6 \\
\hline $\mathrm{C} 7-\mathrm{C} 3-\mathrm{C} 4$ & $121.96(18)$ & $\mathrm{C} 12-\mathrm{C} 13-\mathrm{H} 13 \mathrm{~A}$ & 109.6 \\
\hline $\mathrm{C} 2-\mathrm{C} 3-\mathrm{C} 4$ & $124.64(18)$ & $\mathrm{N} 2-\mathrm{C} 13-\mathrm{H} 13 \mathrm{~B}$ & 109.6 \\
\hline $\mathrm{N} 1-\mathrm{C} 4-\mathrm{C} 3$ & $109.76(17)$ & $\mathrm{C} 12-\mathrm{C} 13-\mathrm{H} 13 \mathrm{~B}$ & 109.6 \\
\hline $\mathrm{N} 1-\mathrm{C} 4-\mathrm{H} 4 \mathrm{~A}$ & 109.7 & $\mathrm{H} 13 \mathrm{~A}-\mathrm{C} 13-\mathrm{H} 13 \mathrm{~B}$ & 108.1 \\
\hline $\mathrm{C} 3-\mathrm{C} 4-\mathrm{H} 4 \mathrm{~A}$ & 109.7 & $\mathrm{C} 15-\mathrm{C} 14-\mathrm{C} 19$ & $120.5(2)$ \\
\hline $\mathrm{N} 1-\mathrm{C} 4-\mathrm{H} 4 \mathrm{~B}$ & 109.7 & $\mathrm{C} 15-\mathrm{C} 14-\mathrm{S} 2$ & $119.55(16)$ \\
\hline $\mathrm{C} 3-\mathrm{C} 4-\mathrm{H} 4 \mathrm{~B}$ & 109.7 & $\mathrm{C} 19-\mathrm{C} 14-\mathrm{S} 2$ & $119.93(18)$ \\
\hline $\mathrm{H} 4 \mathrm{~A}-\mathrm{C} 4-\mathrm{H} 4 \mathrm{~B}$ & 108.2 & $\mathrm{C} 14-\mathrm{C} 15-\mathrm{C} 16$ & $119.7(2)$ \\
\hline $\mathrm{N} 1-\mathrm{C} 5-\mathrm{C} 6$ & $111.90(17)$ & $\mathrm{C} 14-\mathrm{C} 15-\mathrm{H} 15$ & 120.1 \\
\hline $\mathrm{N} 1-\mathrm{C} 5-\mathrm{H} 5 \mathrm{~A}$ & 109.2 & $\mathrm{C} 16-\mathrm{C} 15-\mathrm{H} 15$ & 120.1 \\
\hline $\mathrm{C} 6-\mathrm{C} 5-\mathrm{H} 5 \mathrm{~A}$ & 109.2 & $\mathrm{C} 15-\mathrm{C} 16-\mathrm{C} 17$ & $120.8(2)$ \\
\hline $\mathrm{N} 1-\mathrm{C} 5-\mathrm{H} 5 \mathrm{~B}$ & 109.2 & $\mathrm{C} 15-\mathrm{C} 16-\mathrm{H} 16$ & 119.6 \\
\hline $\mathrm{C} 6-\mathrm{C} 5-\mathrm{H} 5 \mathrm{~B}$ & 109.2 & $\mathrm{C} 17-\mathrm{C} 16-\mathrm{H} 16$ & 119.6 \\
\hline $\mathrm{H} 5 \mathrm{~A}-\mathrm{C} 5-\mathrm{H} 5 \mathrm{~B}$ & 107.9 & $\mathrm{C} 16-\mathrm{C} 17-\mathrm{C} 18$ & $118.5(2)$ \\
\hline $\mathrm{C} 7-\mathrm{C} 6-\mathrm{C} 5$ & $107.88(17)$ & $\mathrm{C} 16-\mathrm{C} 17-\mathrm{C} 20$ & $120.8(2)$ \\
\hline $\mathrm{C} 7-\mathrm{C} 6-\mathrm{H} 6 \mathrm{~A}$ & 110.1 & $\mathrm{C} 18-\mathrm{C} 17-\mathrm{C} 20$ & $120.7(2)$ \\
\hline $\mathrm{C} 5-\mathrm{C} 6-\mathrm{H} 6 \mathrm{~A}$ & 110.1 & $\mathrm{C} 19-\mathrm{C} 18-\mathrm{C} 17$ & $121.5(2)$ \\
\hline $\mathrm{C} 7-\mathrm{C} 6-\mathrm{H} 6 \mathrm{~B}$ & 110.1 & $\mathrm{C} 19-\mathrm{C} 18-\mathrm{H} 18$ & 119.2 \\
\hline $\mathrm{C} 5-\mathrm{C} 6-\mathrm{H} 6 \mathrm{~B}$ & 110.1 & $\mathrm{C} 17-\mathrm{C} 18-\mathrm{H} 18$ & 119.2 \\
\hline $\mathrm{H} 6 \mathrm{~A}-\mathrm{C} 6-\mathrm{H} 6 \mathrm{~B}$ & 108.4 & $\mathrm{C} 18-\mathrm{C} 19-\mathrm{C} 14$ & $118.9(2)$ \\
\hline
\end{tabular}




\begin{tabular}{|c|c|c|c|}
\hline $\mathrm{C} 3-\mathrm{C} 7-\mathrm{C} 6$ & $125.32(19)$ & $\mathrm{C} 18-\mathrm{C} 19-\mathrm{H} 19$ & 120.5 \\
\hline $\mathrm{C} 3-\mathrm{C} 7-\mathrm{S} 1$ & $111.06(16)$ & $\mathrm{C} 14-\mathrm{C} 19-\mathrm{H} 19$ & 120.5 \\
\hline $\mathrm{C} 6-\mathrm{C} 7-\mathrm{S} 1$ & $123.52(15)$ & $\mathrm{C} 17-\mathrm{C} 20-\mathrm{H} 20 \mathrm{~A}$ & 109.5 \\
\hline $\mathrm{O} 1-\mathrm{C} 8-\mathrm{N} 1$ & $122.4(2)$ & $\mathrm{C} 17-\mathrm{C} 20-\mathrm{H} 20 \mathrm{~B}$ & 109.5 \\
\hline $\mathrm{O} 1-\mathrm{C} 8-\mathrm{C} 9$ & $119.81(19)$ & $\mathrm{H} 20 \mathrm{~A}-\mathrm{C} 20-\mathrm{H} 20 \mathrm{~B}$ & 109.5 \\
\hline $\mathrm{N} 1-\mathrm{C} 8-\mathrm{C} 9$ & $117.75(19)$ & $\mathrm{C} 17-\mathrm{C} 20-\mathrm{H} 20 \mathrm{C}$ & 109.5 \\
\hline $\mathrm{N} 2-\mathrm{C} 9-\mathrm{C} 8$ & $113.56(17)$ & $\mathrm{H} 20 \mathrm{~A}-\mathrm{C} 20-\mathrm{H} 20 \mathrm{C}$ & 109.5 \\
\hline $\mathrm{N} 2-\mathrm{C} 9-\mathrm{H} 9 \mathrm{~A}$ & 108.9 & $\mathrm{H} 20 \mathrm{~B}-\mathrm{C} 20-\mathrm{H} 20 \mathrm{C}$ & 109.5 \\
\hline $\mathrm{C} 8-\mathrm{C} 9-\mathrm{H} 9 \mathrm{~A}$ & 108.9 & & \\
\hline $\mathrm{O} 3-\mathrm{S} 2-\mathrm{N} 3-\mathrm{C} 12$ & $43.28(18)$ & $\mathrm{C} 10-\mathrm{N} 2-\mathrm{C} 9-\mathrm{C} 8$ & $-71.2(2)$ \\
\hline $\mathrm{O} 2-\mathrm{S} 2-\mathrm{N} 3-\mathrm{C} 12$ & $172.33(16)$ & $\mathrm{O} 1-\mathrm{C} 8-\mathrm{C} 9-\mathrm{N} 2$ & $112.0(2)$ \\
\hline $\mathrm{C} 14-\mathrm{S} 2-\mathrm{N} 3-\mathrm{C} 12$ & $-72.46(17)$ & $\mathrm{N} 1-\mathrm{C} 8-\mathrm{C} 9-\mathrm{N} 2$ & $-70.2(2)$ \\
\hline $\mathrm{O} 3-\mathrm{S} 2-\mathrm{N} 3-\mathrm{C} 11$ & $178.81(15)$ & $\mathrm{C} 9-\mathrm{N} 2-\mathrm{C} 10-\mathrm{C} 11$ & $-179.18(16)$ \\
\hline $\mathrm{O} 2-\mathrm{S} 2-\mathrm{N} 3-\mathrm{C} 11$ & $-52.14(17)$ & $\mathrm{C} 13-\mathrm{N} 2-\mathrm{C} 10-\mathrm{C} 11$ & $-59.1(2)$ \\
\hline $\mathrm{C} 14-\mathrm{S} 2-\mathrm{N} 3-\mathrm{C} 11$ & $63.07(16)$ & $\mathrm{C} 12-\mathrm{N} 3-\mathrm{C} 11-\mathrm{C} 10$ & $-56.6(2)$ \\
\hline $\mathrm{C} 7-\mathrm{S} 1-\mathrm{C} 1-\mathrm{C} 2$ & $-1.04(18)$ & $\mathrm{S} 2-\mathrm{N} 3-\mathrm{C} 11-\mathrm{C} 10$ & $165.84(13)$ \\
\hline $\mathrm{S} 1-\mathrm{C} 1-\mathrm{C} 2-\mathrm{C} 3$ & $1.2(2)$ & $\mathrm{N} 2-\mathrm{C} 10-\mathrm{C} 11-\mathrm{N} 3$ & $56.3(2)$ \\
\hline $\mathrm{C} 1-\mathrm{C} 2-\mathrm{C} 3-\mathrm{C} 7$ & $-0.7(3)$ & $\mathrm{C} 11-\mathrm{N} 3-\mathrm{C} 12-\mathrm{C} 13$ & $58.5(2)$ \\
\hline $\mathrm{C} 1-\mathrm{C} 2-\mathrm{C} 3-\mathrm{C} 4$ & $178.0(2)$ & $\mathrm{S} 2-\mathrm{N} 3-\mathrm{C} 12-\mathrm{C} 13$ & $-163.93(14)$ \\
\hline $\mathrm{C} 8-\mathrm{N} 1-\mathrm{C} 4-\mathrm{C} 3$ & $129.51(19)$ & $\mathrm{C} 9-\mathrm{N} 2-\mathrm{C} 13-\mathrm{C} 12$ & $-177.01(17)$ \\
\hline $\mathrm{C} 5-\mathrm{N} 1-\mathrm{C} 4-\mathrm{C} 3$ & $-45.7(2)$ & $\mathrm{C} 10-\mathrm{N} 2-\mathrm{C} 13-\mathrm{C} 12$ & $61.5(2)$ \\
\hline $\mathrm{C} 7-\mathrm{C} 3-\mathrm{C} 4-\mathrm{N} 1$ & $15.1(3)$ & $\mathrm{N} 3-\mathrm{C} 12-\mathrm{C} 13-\mathrm{N} 2$ & $-60.7(2)$ \\
\hline $\mathrm{C} 2-\mathrm{C} 3-\mathrm{C} 4-\mathrm{N} 1$ & $-163.44(19)$ & $\mathrm{O} 3-\mathrm{S} 2-\mathrm{C} 14-\mathrm{C} 15$ & $140.22(17)$ \\
\hline $\mathrm{C} 8-\mathrm{N} 1-\mathrm{C} 5-\mathrm{C} 6$ & $-110.0(2)$ & $\mathrm{O} 2-\mathrm{S} 2-\mathrm{C} 14-\mathrm{C} 15$ & $9.6(2)$ \\
\hline $\mathrm{C} 4-\mathrm{N} 1-\mathrm{C} 5-\mathrm{C} 6$ & $64.8(2)$ & $\mathrm{N} 3-\mathrm{S} 2-\mathrm{C} 14-\mathrm{C} 15$ & $-105.18(18)$ \\
\hline $\mathrm{N} 1-\mathrm{C} 5-\mathrm{C} 6-\mathrm{C} 7$ & $-46.2(2)$ & $\mathrm{O} 3-\mathrm{S} 2-\mathrm{C} 14-\mathrm{C} 19$ & $-37.9(2)$ \\
\hline $\mathrm{C} 2-\mathrm{C} 3-\mathrm{C} 7-\mathrm{C} 6$ & $176.41(19)$ & $\mathrm{O} 2-\mathrm{S} 2-\mathrm{C} 14-\mathrm{C} 19$ & $-168.58(16)$ \\
\hline $\mathrm{C} 4-\mathrm{C} 3-\mathrm{C} 7-\mathrm{C} 6$ & $-2.3(3)$ & N3-S2-C14-C19 & $76.67(18)$ \\
\hline $\mathrm{C} 2-\mathrm{C} 3-\mathrm{C} 7-\mathrm{S} 1$ & $-0.1(2)$ & $\mathrm{C} 19-\mathrm{C} 14-\mathrm{C} 15-\mathrm{C} 16$ & $1.4(3)$ \\
\hline $\mathrm{C} 4-\mathrm{C} 3-\mathrm{C} 7-\mathrm{S} 1$ & $-178.81(16)$ & $\mathrm{S} 2-\mathrm{C} 14-\mathrm{C} 15-\mathrm{C} 16$ & $-176.74(16)$ \\
\hline $\mathrm{C} 5-\mathrm{C} 6-\mathrm{C} 7-\mathrm{C} 3$ & $17.4(3)$ & $\mathrm{C} 14-\mathrm{C} 15-\mathrm{C} 16-\mathrm{C} 17$ & $-0.7(3)$ \\
\hline $\mathrm{C} 5-\mathrm{C} 6-\mathrm{C} 7-\mathrm{S} 1$ & $-166.53(15)$ & $\mathrm{C} 15-\mathrm{C} 16-\mathrm{C} 17-\mathrm{C} 18$ & $-0.8(3)$ \\
\hline $\mathrm{C} 1-\mathrm{S} 1-\mathrm{C} 7-\mathrm{C} 3$ & $0.62(17)$ & $\mathrm{C} 15-\mathrm{C} 16-\mathrm{C} 17-\mathrm{C} 20$ & $178.8(2)$ \\
\hline $\mathrm{C} 1-\mathrm{S} 1-\mathrm{C} 7-\mathrm{C} 6$ & $-175.95(18)$ & $\mathrm{C} 16-\mathrm{C} 17-\mathrm{C} 18-\mathrm{C} 19$ & $1.6(3)$ \\
\hline $\mathrm{C} 4-\mathrm{N} 1-\mathrm{C} 8-\mathrm{O} 1$ & $2.8(3)$ & $\mathrm{C} 20-\mathrm{C} 17-\mathrm{C} 18-\mathrm{C} 19$ & $-178.0(2)$ \\
\hline $\mathrm{C} 5-\mathrm{N} 1-\mathrm{C} 8-\mathrm{O} 1$ & $177.38(19)$ & $\mathrm{C} 17-\mathrm{C} 18-\mathrm{C} 19-\mathrm{C} 14$ & $-0.9(3)$ \\
\hline $\mathrm{C} 4-\mathrm{N} 1-\mathrm{C} 8-\mathrm{C} 9$ & $-174.92(17)$ & $\mathrm{C} 15-\mathrm{C} 14-\mathrm{C} 19-\mathrm{C} 18$ & $-0.6(3)$ \\
\hline $\mathrm{C} 5-\mathrm{N} 1-\mathrm{C} 8-\mathrm{C} 9$ & $-0.4(3)$ & $\mathrm{S} 2-\mathrm{C} 14-\mathrm{C} 19-\mathrm{C} 18$ & $177.51(16)$ \\
\hline $\mathrm{C} 13-\mathrm{N} 2-\mathrm{C} 9-\mathrm{C} 8$ & $168.59(18)$ & & \\
\hline
\end{tabular}

Hydrogen-bond geometry $\left(A,{ }^{\circ}\right)$

\begin{tabular}{lllll}
\hline$D-\mathrm{H} \cdots A$ & $D-\mathrm{H}$ & $\mathrm{H} \cdots A$ & $D \cdots A$ & $D-\mathrm{H} \cdots A$ \\
\hline $\mathrm{C} 5-\mathrm{H} 5 A \cdots \mathrm{S} 1^{\mathrm{i}}$ & 0.99 & 2.77 & $3.469(2)$ & 128
\end{tabular}


supporting information

$\begin{array}{llllr}\mathrm{C} 6-\mathrm{H} 6 A \cdots \mathrm{O} 1^{\mathrm{ii}} & 0.99 & 2.52 & 3.470(3) & 161 \\ \mathrm{C} 6-\mathrm{H} 6 B \cdots \mathrm{O} 2^{\mathrm{iii}} & 0.99 & 2.51 & 3.346(3) & 143\end{array}$

Symmetry codes: (i) $x+1 / 2,-y+3 / 2,-z+1$; (ii) $-x,-y+2,-z+1$; (iii) $-x+1 / 2, y-1 / 2, z$. 